\title{
MIBG and imaging of cardiac adrenergic system: From heart failure to ventricular arrhythmias and atrial fibrillation, through cardiac asynchrony. What else?
}

\author{
Claudio Marcassa, $\mathrm{MD}^{\mathrm{a}}$ \\ a Cardiology Department, Maugeri Clinical and Scientific Institutes, IRCCS, Scientific Institute of \\ Veruno (NO), Veruno, Italy
}

Received Jun 2, 2021; accepted Jun 3, 2021

doi: $10.1007 / \mathrm{s} 12350-021-02699-3$

See related article, pp. 2220-2231

The role of the autonomic nervous system in the pathogenesis of ventricular arrhythmias and sudden cardiac death is well documented. ${ }^{1-3}$

Since the first report on the imaging of cardiac neuronal damage by $123 \mathrm{I}-\mathrm{mIBG}$ late in the 80 ', the evaluation of the integrity of the cardiac autonomic nervous system and its relation with myocardial perfusion and function has been documented in a wide clinical scenarios.

In early canine experimental studies, it was documented that myocardial ischemia induces an area of neuronal damage larger than the ischemic or necrotic area. ${ }^{4-7}$ This viable, denervated, myocardium is affected by a sympathetic denervation supersensitivity that makes the myocardium prone to ventricular fibrillation during the infusion of norepinephrine compared with the control group. ${ }^{4}$

In a small clinical study in patients with recent myocardial infarction, Stanton et al documented that 10 (83\%) of the 12 postinfarction patients with ventricular arrhythmias had areas of 123I-mIBG/thallium-201 mismatch, but only 2 (29\%) of the 7 patients without ventricular arrhythmias demonstrated 123I-mIBG /thallium-201 mismatch. ${ }^{8}$

\footnotetext{
Reprint requests: Claudio Marcassa, MD, Cardiology Department, Maugeri Clinical and Scientific Institutes, IRCCS, Scientific Institute of Veruno (NO), Via Per Revislate 13, 28010 Veruno, Italy; claudio.marcassa@icsmaugeri.it

J Nucl Cardiol 2022;29:2232-4.

$1071-3581 / \$ 34.00$

Copyright (c) 2021 American Society of Nuclear Cardiology.
}

Areas of mismatch were also documented in patients with idiopathic dilated cardiomyopathy, and the $\mathrm{H} / \mathrm{M}$ was inversely correlated with left ventricular ejection fraction ${ }^{9}$; in a more recent study, Marini et al. concluded that perfusion defects seem to differently affect 123I-mIBG retention in heart failure caused by ischemic heart disease or dilated cardiomyopathy. ${ }^{10}$

Several studies have demonstrated the usefulness of 123I-mIBGs to identify patients with a deregulated autonomic nervous system and a mismatch with regional perfusion that may predispose to cardiac electrical instability and, finally, to a high risk of malignant arrhythmias, ${ }^{11-13}$ mainly in patients with ischemic heart disease.

In the field of congestive heart failure, several single center experiences or retrospective studies initially suggested the prognostic value of cardiac 123I-mIBG imaging. The multicenter ADMIRE-HF study, the larger study so far, prospectively enrolled 964 patients with left ventricular dysfunction and heart failure and documented the higher event rate, sudden arrhythmic death, or death due to worsening heart failure, in patients with a $\mathrm{H} / \mathrm{M}$ ratio $<1.6 .^{14}$

Available studies, reviews and meta-analysis, thus strongly indicate that $123 \mathrm{I}-\mathrm{mIBGs}$ imaging has the capability to identify patients at high risk of MACE (death, malignant ventricular arrhythmias) or worsening heart failure and those with a more favorable prognosis, due to the very high negative predictive value, and with a prognostic impact that is incremental over clinical information alone (e.g., left ventricular ejection fraction and BNP).

Moreover, Boogers et al ${ }^{15}$ documented in 116 patients with ICD implanted for primary or secondary prevention, that 123I-mIBG SPECT defect score (with a 
summed score greater than 26) was an independent predictor of appropriated ICD therapy.

The prognostic values of late $\mathrm{H} / \mathrm{M}$ and wash-out rate were also documented in patients with heart failure and preserved left ventricular ejection fraction (HFpEF). ${ }^{16}$ Last but not least myocardial sympathetic denervation, evaluated by $123 \mathrm{I}-\mathrm{mIBG}$ imaging, matched with the perfusion pattern and was also linked to regional asynchrony, in particular with the most dyssynchronous walls. 17,18

In this issue of the journal, Sazanova et al ${ }^{19}$ used adrenergic imaging to predict outcome in 82 patients with atrial fibrillation (AF) undergoing catheter ablation procedure; they concluded that $123 \mathrm{I}-\mathrm{mIBG}$ imaging data (H/M and WO) performed before ablation were independently associated, at multivariable analysis, to $\mathrm{AF}$ relapse at follow-up, with an AUC at ROC analysis of 0.79 and 0.71 , respectively.

This is a relatively new field of investigation for 123I-mIBG imaging. The correlation between cardiac autonomic nervous system and AF occurrence and maintenance has been documented by several authors. $^{20-23}$ In patients with AF, 123I-mIBG imaging has been classically performed to predict hard events: myocardial infarction and heart failure. ${ }^{20-24}$

Recently, several authors investigated the possible link between 123I-mIBG imaging performed before ablation and the outcome, with discordant results. ${ }^{22-24}$

Discrepancies between the manuscript by Sazanova et al and other studies have been discussed in depth and mainly ascribed to the possible presence of patients with latent $\mathrm{HFpEF}$ (thus prone to sympathetic system derangement) or to differences in imaging modalities (e.g., different collimator use, with different values for $\mathrm{H} / \mathrm{M}$ and wash-out rate).

Recently, solid-state CZT SPECT was used in a preliminary study to assess the adrenergic innervation of the left atrium that actually is the target of ablation procedure. $^{25}$

\section{CONCLUSION}

Since its first use late in the 80', a lot of studies documented that 123I-mIBG and Imaging of Cardiac Adrenergic System progressively extended and demonstrated its value in a wide clinical scenario: from heart failure and myocardial infarction, including cardiac asynchrony, to ventricular arrhythmias and sudden death prediction to atrial fibrillation. Furthermore, myocardial innervation can only be assessed by nuclear techniques.

From the analysis of available literature, major limitations can still be identified in a widespread utilization of the techniques to have a potential impact on the clinical decision making: a real standardization of the cut-off used for prognostication, either H/M, Washout or summed scores, in different settings, and according to the different acquisition modalities, and a more diffuse utilization of solid-state cameras. Larger multicenter studies in the different clinical scenario are needed.

After that, what else? Hopefully, perhaps, to convince cardiologist.

\section{Conflict of interest}

None declared

\section{References}

1. Jacobson AF, Senior R, Cerqueira MD, Wong N, Thomas G, Lopez V, et al. Myocardial Iodine-123 meta-iodobenzylguanidine imaging and cardiac events in heart failure. Results of the prospective ADMIRE-HF (AdreView myocardial imaging for risk evaluation in heart failure) study. J Am Coll Cardiol 2010;55:2212-21

2. Barron HV, Lesh MD. Autonomic nervous system and sudden cardiac death. J Am Coll Cardiol 1996;27:1053-60.

3. Zipes DP. Sympathetic stimulation and arrhythmias. N Engl J Med 1991;325:656-7.

4. Inoue H, Zipes DP. Results of sympathetic denervation in the canine heart: supersensitivity that may be arrhythmogenic. Circulation 1987;75:877-87.

5. Inoue H, Skale BT, Zipes DP. Effects of ischemia on cardiac afferent sympathetic and vagal reflexes in dog. Am $\mathrm{J}$ Physiol 1988;255:H26-35.

6. Inoue H, Zipes DP. Time course of denervation of efferent sympathetic and vagal nerves after occlusion of the coronary artery in the canine heart. Circ Res 1988;62:1111-20.

7. Kammerling JJ, Green FJ, Watanabe AM, Inoue H, Barber MJ, Henry DP, et al. Denervation supersensitivity of refractoriness in noninfarcted areas apical to transmural myocardial infarction. Circulation 1987;76:383-93.

8. Stanton MS, Tuli MM, Radtke NL, Heger JJ, Miles WM, Mock $\mathrm{BH}$, et al. Regional sympathetic denervation after myocardial infarction in humans detected noninvasively using I-123metaiodobenzylguanidine. J Am Coll Cardiol 1989;14:1519-26.

9. Schofer J, Spielmann R, Schuchert A, Weber K, Schlüter M. Iodine-123 meta-iodobenzylguanidine scintigraphy: a noninvasive method to demonstrate myocardial adrenergic nervous system disintegrity in patients with idiopathic dilated cardiomyopathy. $\mathrm{J}$ Am Coll Cardiol 1988;12:1252-8.

10. Marini C, Giorgetti A, Gimelli A, Kusch A, Sereni N, L'abbate A, et al. Extension of myocardial necrosis differently affects MIBG retention in heart failure caused by ischaemic heart disease or by dilated cardiomyopathy. Eur $\mathrm{J}$ Nucl Med Mol Imaging 2005;32:682-8.

11. Verberne HJ, Brewster LM, Somsen GA, van Eck-Smit B. Prognostic value of myocardial 123I-metaiodobenzylguanidine (MIBG) parameters in patients with heart failure: a systematic review. Eur Heart J 2008;29:1147-59.

12. Merlet P, Benvenuti C, Moyse D, Pouillart F, Dubois-Randè JL, Duval AM, et al. Prognostic value of MIBG imaging in idiopathic dilated cardiomyopathy. J Nucl Med 1999;40:917-23. 
13. Hattori N, Schwaiger M. metaiodobenzylguanidine scintigraphy of the heart: what have we learnt clinically? Eur J Nucl Med 2000;27:1-6.

14. Jacobson AF, Senior R, Cerqueira MD, Wong ND, Thomas GS, Lopez VA, et al. ADMIRE-HF Investigators. Myocardial iodine123 meta-iodobenzylguanidine imaging and cardiac events in heart failure Results of the prospective ADMIRE-HF (AdreView Myocardial Imaging for Risk Evaluation in Heart Failure) study. J Am Coll Cardiol 2010;55:2212-21.

15. Boogers MJ, Borleffs CJ, Henneman MM, van Bommel RJ, van Ramshorst J, Boersma E, et al. Cardiac sympathetic denervation assessed with 123-iodine metaiodobenzylguanidine imaging predicts ventricular arrhythmias in implantable cardioverterdefibrillator patients. J Am Coll Cardiol 2010;55:2769-77.

16. Katoh S, Shishido T, Kutsuzawa D, Arimoto T, Netsu S, Funayama A, et al. Iodine-123-metaiodobenzylguanidine imaging can predict future cardiac events in heart failure patients with preserved ejection fraction. Ann Nucl Med 2010;24:679-86.

17. Schlack W, Thämer V. Unilateral changes of sympathetic tone to the heart impair left ventricular function. Acta Anaesthesiol Scand 1996;40:262-71.

18. Gimelli A, Liga R, Menichetti F, Soldati E, Bongiorni MG, Marzullo P. Interactions between myocardial sympathetic denervation and left ventricular mechanical dyssynchrony: A CZT analysis. J Nucl Cardiol 2017. https://doi.org/10.1007/s12350-0171036-3.

19. Sazonova SI, Varlamova JV, Nikitin NA, Minin SM, Kisteneva IV, Batalov RE, et al. J Nucl cardiol; in press.
20. Akutsu Y, Kaneko K, Kodama Y, Li H-L, Suyama J, Shinozuka A, et al. Significance of cardiac sympathetic nervous system abnormality for predicting vascular events in patients with idiopathic paroxysmal atrial fibrillation. Eur J Nucl Med Mol Imaging 2010;37:742-9.

21. Akutsu Y, Kaneko K, Kodama Y, Li H-L, Suyama J, Shinozuka A, et al. Iodine-123 mIBG imaging for predicting the development of atrial fibrillation. JACC Cardiovasc Imaging 2011;4:78-86.

22. Arimoto T, Tada H, Igarashi M, Sekiguchi Y, Sato A, Koyama T, et al. High washout rate of iodine-123-metaiodobenzylguanidine imaging predicts the outcome of catheter ablation of atrial fibrillation. J Cardiovasc Electrophysiol 2011;22:1297-304.

23. Wenning C, Lange PS, Schulke C, Vrachimis A, Monnig G, Schober $\mathrm{O}$, et al. Pulmonary vein isolation in patients with paroxysmal atrial fibrillation is associated with regional cardiac sympathetic denervation. EJNMMI Res 2013;3:81.

24. Lemery R, Ben-Haim S, Wells G, Ruddy TD. I-123-Metaiodobenzylguanidine imaging in patients with atrial fibrillation undergoing cardiac mapping and ablation of autonomic ganglia. Heart Rhythm 2017;14:128-32.

25. Stirrup J, Gregg S, Baavour R, Roth N, Breault C, Agostini D, et al. Hybrid solid-state SPECT/CT left atrial innervation imaging for identification of left atrial ganglionated plexi: Technique and validation in patients with atrial fibrillation. J Nucl Cardiol 2020;27:1939-50.

Publisher's Note Springer Nature remains neutral with regard to jurisdictional claims in published maps and institutional affiliations. 